\title{
Improved Certificate-Based Encryption Scheme in the Big Data: Combining AES and (ECDSA - ECDH)
}

\author{
Omar Salah F. Shareef \\ omar.alshareef@uofallujah.edu.iq \\ University of Fallujah, Fallujah, Iraq
}

\author{
Ali Makki Sagheer \\ prof.ali@alqalam.edu.iq \\ Qalam University College, Kirkuk, Iraq
}

\begin{abstract}
Big data usually running in large-scale and centralized key management systems. However, the centralized key management systems are increasing the problems such as single point of failure, exchanging a secret key over insecure channels, third-party query, and key escrow problem. To avoid these problems, we propose an improved certificatebased encryption scheme that ensures data confidentiality by combining symmetric and asymmetric cryptography schemes. The combination can be implemented by using the Advanced Encryption Standard (AES) and Elliptic Curve Diffie-Hellman (ECDH). The proposed scheme is an enhanced version of the Certificate-Based Encryption (CBE) scheme and preserves all its advantages. However, the key generation process in our scheme has been done without any intervention from the certificate issuer and avoiding the risk of compromised CA. The Elliptic Curve Digital Signature Algorithm (ECDSA) has been used with the ECDH to handle the authentication of the key exchange. The proposed scheme is demonstrated on a big dataset of social networks. The scheme is analyzed based on security criteria that have been compared with the previous schemes to evaluate its performance.
\end{abstract}

Keywords: Big Data Security; Certificate-Based Encryption; ECDSA; ECDH; AES.

\section{Introduction}

The term 'Big Data' has been become an essential part of people's lives. The appearance of big data has brought new challenges regarding data security. The collecting, storage, manipulation and retention of huge quantities of data have led to critical security and privacy considerations (Cheng et al.,2017). Accessing the data needs to be controlled to affirm that the non-eligible entities cannot tamper with or access the data. Improving the security and authentication of sensitive data can give the companies new businesses opportunities. Therefore, close attention has been drawn to secure data firmly from any unauthorized access. The main challenge in big data that are using a complicated distributed system, is the multifaceted nature of overseeing wide usage. Verification ought to be overseen by an adaptable, hearty and versatile framework that denies a malevolent client from access big data. Accordingly, new ways to deal with security are required to defeat the security blemishes in the current usage. However, there are two methods to design a secure system and protecting the privacy of the receivers in a communication system; symmetric and asymmetric keys.

The secure cryptosystem is based on effective key management. However, the security of any cryptosystem is dependent upon how securely its keys are managed?

Public Key Infrastructure (PKI) generates and handles a pair of keys in addition to the certificate. The main challenge of managing the digital certificate in public key infrastructure is when we have many nodes in a distributed environment. 
To cope with this issue, Shamir (A Shamir, 1984) produced the concept of Identity-Based Encryption (IBE) where the identity was used as a public key, and a third party generates a secret key for the user. This approach eliminates the demand for digital certificates. However, IBE suffers from a key escrow problem, the third party holds the private keys of clients, as a result, it can peek at all communication data of users and successfully disguise himself as any user to sign a message. Alternatively, Certificate-Based Encryption (CBE) overcomes the drawbacks of PKI and IBE.

In 2003, Gentry (Gentry, 2003) presented the idea of CBE. This scheme merges PublicKey Encryption (PKE) and IBE while safeguarding the highlights of schemes. As in conventional PKE, every client produces a pair of keys then asks for a certificate from the Certificate Authority (CA). The significant matter in CBE is a certificate works as a partial private key as well as its work as a certificate. This function produces an effective implicit certificate, thus a recipient wants the certificate together with his/her private key to decrypt a ciphertext, whilst the senders do not need to be concerned about the certificate revocation problem. However, the CBE copes with the limitations of IBE and PKE. The CBE can ignore third-party queries. Then, disregarding a secure channel among the CA and clients. Furthermore, it is avoiding the key escrow problem (since CA does not know the private keys of users). Finally, it fixes the certificate revocation problem.

In recent years, $\mathrm{CBE}$ has attracted considerable interest in the community of research and several schemes in CBE have been proposed (Liu and Zhou, 2008; Lu et al., 2008; Galindo et al., 2008; Hyla et al., 2015; Lu and Li, 2014; Le, Kim and Hwang, 2016; Hwang and Le, 2018).

In this paper, we propose an efficient certificate-based encryption scheme that ensures data confidentiality by combining symmetric and asymmetric cryptography schemes. Particularly, using the Advanced Encryption Standard (AES) and Elliptic Curve DiffieHellman (ECDH). Moreover, the Elliptic Curve Digital Signature Algorithm (ECDSA) has been used with ECDH to handle the weakness of authentication between the nodes whilst using the ECDH approach. When the system is on a large scale, the task will be computationally intensive. Moreover, the proposed scheme can be supported distributive processing in large-scale key management within a distributed environment.

\section{PRELIMINARIES}

\subsection{ECDSA}

ECDSA is the elliptic curve analog of the Digital Signature Algorithm DSA (Johnson et al., 2001). Vanstone proposed the ECDSA scheme as an answer to the request of the National Institute of Standards and Technology (NIST) for feedback on their proposal about the Digital Signature Scheme (DSS) (S. Vanstone, 1992).

DSS is similar to handwritten signatures. The digital signature represents a number based on the private key known only by the signer and on the contents of the signed message, as well as, the signatures should be verified without gain access to the secret key of the signer (Langford, 1995). ECDSA algorithm is defined as follows:

\section{Setup}

1. Alice and $\mathrm{Bob}$ select $\mathrm{a}$ finite field $\mathrm{Fq}$ and an elliptic curve E over $\mathrm{Fq}(\mathrm{E}(\mathrm{Fq}))$.

2. They choose a random base point $B \in E$ with order $n$, such that $B$ generates a large subgroup of E, preferably of the same order as that of E itself.

For more information about the Conference please visit the websites:
http://ihicps.com/
C o m p u t e r $\mid 83$ 


\section{Key generation}

1. choose a secret random integer $d$ in the interval $[2, N]$

2. Computes $\mathrm{Q}=\mathrm{dB}$

3. Make $Q$ public and keep d secret

\section{Signature generation}

Alice sends the signed message to Bob as follows

1. Select random integer $\mathrm{k}$ in the interval $[2, \mathrm{~N}]$

2. Compute $(x, y)=k B$

3. Compute $r=x \bmod n$

4. Compute $\mathrm{e}=\mathrm{H}(\mathrm{M})$

5. Compute $s=k^{-1}(e+d r) \bmod n$

6. The signature for $M$ is $(r, s)$

\section{Signature verification}

Bob verifies Alice's signature $(r, s)$ on message $M$ as follows

1. Compute $\mathrm{e}=\mathrm{H}(\mathrm{M})$

2. Compute $\mathrm{w}=\mathrm{s}^{-1} \bmod \mathrm{n}$

3. Compute $\mathrm{u}_{1}=\mathrm{e} \mathrm{w} \bmod \mathrm{n}$

4. Compute $\mathrm{u}_{2}=\mathrm{rw} \bmod \mathrm{n}$

5. Compute $(x, y)=u_{1} B+u_{2} Q$. If $(x, y)=0$ then reject the signature

6. Otherwise, Compute $v=x \bmod n$

7. Accept the signature if and only if $v=r$

\subsection{ECDH}

ECDH is a key agreement algorithm that lets two entities generate a shared secret key based on ECC (Anoop, 2001). The following example illustrates how the key establishment will be made.

Suppose A wants to generate a shared key with B through a channel that may be eavesdropped on by a third party. Initially, each entity should agree upon Elliptic Curve domain parameters (q, a, b, G, n, h). Each has a pair of keys; $d$ is a secret key (which is a random integer $<n$, where $n$ is the order of the curve, a domain parameter of the elliptic curve) and a public key $Q=d * G$ ( where $\mathrm{G}$ is the generator factor, a domain parameter 
of the elliptic curve). Suppose $d_{A} \cdot Q_{A}$ are the secret/public key of $\mathrm{A}$, and $d_{B} \cdot Q_{B}$ are the secret/public key of B.

1. A computes $\mathrm{E}=\left(X_{E}, Y_{E}\right)=d_{A} \cdot Q_{B}$

2. B computes $\mathrm{F}=\left(X_{F}, Y_{F}\right)=d_{B} \cdot Q_{A}$

3. Since $d_{A} \cdot Q_{B}=d_{A} \cdot d_{B} \cdot G=d_{B} \cdot d_{A} \cdot G=D_{B} \cdot Q_{A}$

4. Thus $\mathrm{E}=\mathrm{F}$ and subsequently $X_{E}=X_{F}$

5. As a result, the shared secret is $X_{E}$

Because of difficulties regarding discovering the secret key $d_{A}$ or $d_{B}$ from the public key $\mathrm{E}$ or F, it's difficult to get the shared secret for an outsider.

The algorithm of ECDH Key Exchange is described as following:

Goal: generate a secure shared key

Input: elliptic curve parameter domain

Output: secure shared key E

Step 1:

1. Client A chooses secret random number $\mathrm{X}<\mathrm{n}$

2. Client $\mathrm{B}$ chooses the secret random number $\mathrm{Y}<\mathrm{n}$

Step 2:

1. Client A computes $P U_{A}=X * G$

2. Client B computes $P U_{B}=Y * G$

The two parties share their public keys and the common base point $G$ Step 3:

1. Client A compute $\mathrm{E}=\mathrm{X} * \mathrm{PU} \mathrm{B}_{\mathrm{B}}$

2. Client $\mathrm{B}$ compute $\mathrm{E}=\mathrm{Y} * \mathrm{PU} \mathrm{A}_{\mathrm{A}}$

Step 4: Return (E)

\section{The Proposed Scheme}

In this research, an improved certificate-based encryption scheme has been proposed, and that ensures data confidentiality by combining symmetric and asymmetric cryptography schemes. Particularly, using the Advanced Encryption Standard (AES) and Elliptic Curve Diffie-Hellman (ECDH). Moreover, the Elliptic Curve Digital Signature Algorithm (ECDSA) was used with ECDH to handle the weakness of authentication between nodes while using the ECDH approach. When the system is on a large scale, the task will be computationally intensive.

The proposed scheme will be reduced the workload of the CA by delegating the task of CA to the user level. In other words, the key generation process is done without any intervention from the certificate provider. The keys are generated using the ECDH algorithm and it's derived from the parameters that generate the keys of the certificate and hence avoiding the risk of compromised CA. Accordingly, it can also resolve the problem of violation of any privacy of customers because it resolves the key escrow problem. For more information about the Conference please visit the websites: 
Moreover, the proposed scheme can be supported distributive processing in large-scale key management within a distributed environment. The overview of system layers is shown in more detail in Figure 1, which presents the block diagram of system operations using the improved CBE.

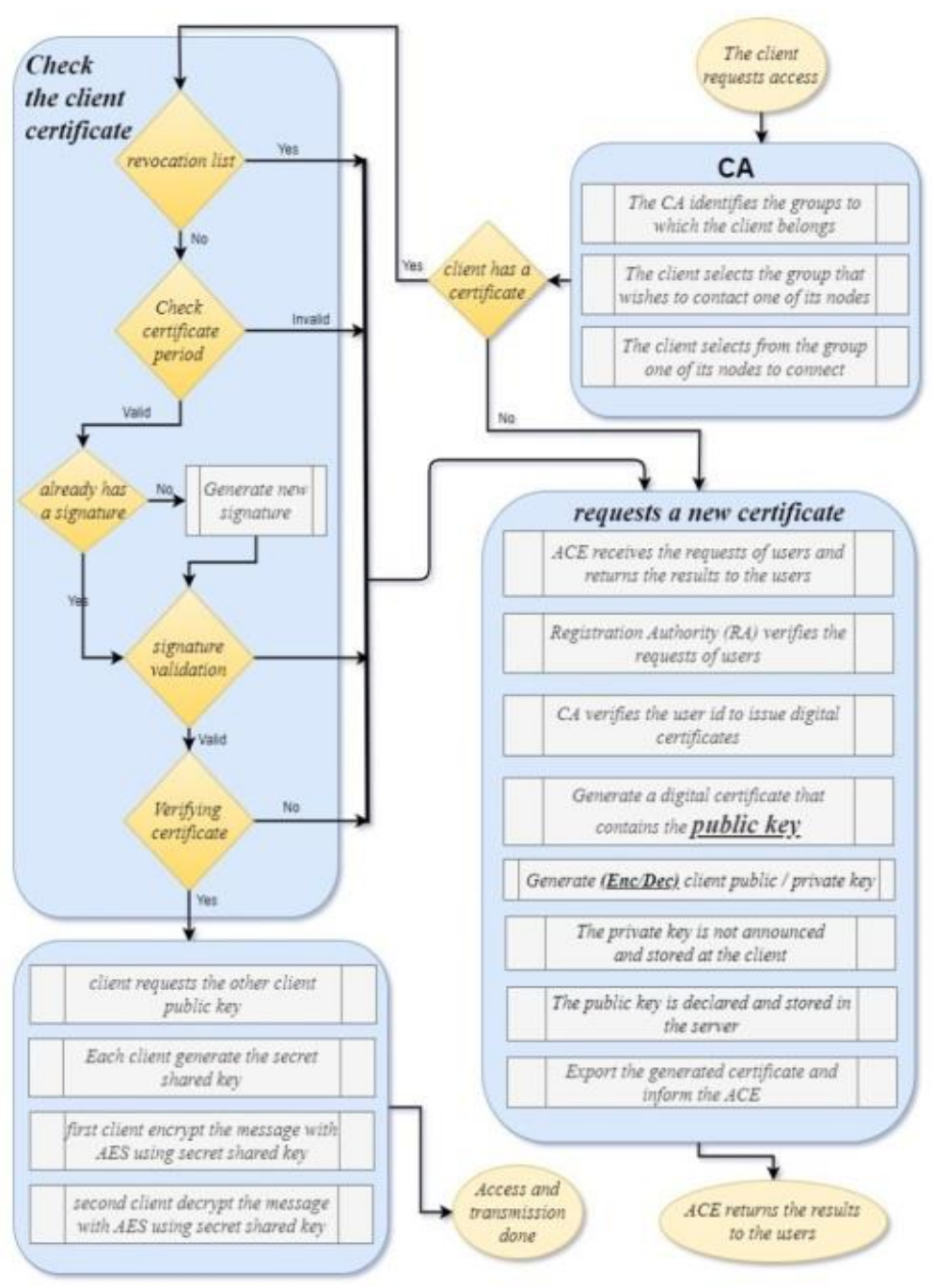

Figure 1 .The Block Diagram of the Proposed Model

By utilizing the benefit of ECDSA, our proposed scheme was built by applying the ECDSA with ECDH that generating the encryption keys. In particular, we designed the setup and certification algorithm by utilizing the key generation as well as the signature generation algorithms of ECDSA. Additionally, by using the key generation algorithm of ECDH, we design the SetKeyPair algorithm. Symmetric algorithm AES is used for

For more information about the Conference please visit the websites:
http://ihicps.com/
C o m p u t e r $\mid 86$ 
constructing encryption and decryption algorithms, in this research AES-128 is used. ECDSA and ECDH are based on some parameters for all network participants.

which:

$\mathrm{q}=$ Order of the prime field $\mathrm{Fq}$

$\mathrm{E}=$ an elliptic curve $\mathrm{y} 2=\mathrm{x} 3+\mathrm{ax}+\mathrm{b}$ defined over the prime field $\mathrm{Fq}$

$\mathrm{G}=\mathrm{A}$ random non-zero base point in $\mathrm{E}(\mathrm{Fq})$

$\mathrm{n}=$ the order of $\mathrm{G}$, typically a prime

$\mathrm{h}=$ the cofactor $\frac{|\mathrm{E}(\mathrm{fq})|}{\mathrm{n}}$

The improved CBE scheme consists of six algorithms:

\section{Setup:}

ECDSA and ECDH are based on some parameters (params) common for all network participants. Setup run by the CA. It takes the security parameter as input and returns the CA's master secret key msk.

\section{Certificate SetKeyPair:}

Input: ID, Domain Parameters.

Output: private key $d$ and secret key $Q$ and master secret key E for encryption/decryption. private key: $d \in R[1, n-1]$ public key: $Q=d G \in E(F q)$

\section{Encryption/Decryption SetKeyPair:}

This process has been performed by users without the intervention of the certificate provider. The keys that have been generated will be using the same parameters that are used to generate certificate keys. The shared key is generated using ECDH.

\section{Certification:}

ECDSA takes ID, params, $m s k, Q$ and additional identifying information, such as name, as input, and the output is the certificate Cert to the user.

\section{A. ECDSA Signature Generation}

Input: Domain Parameters, the secret key of the signer $d$ and message $M$

Output: ECDSA signature $(r, s)$

$\mathrm{k}=\mathrm{a}$ haphazardly picked component in $[1, n-1]$ (the session key)

$R=k G$

$r=x(R)$ (the $\mathrm{x}$-coordinate of $\mathrm{R}$ ) reduced module $n$

$s=k=1(H(M)+d r) \bmod n, \mathrm{H}$ is a cryptographic hash function

\section{B. ECDSA Signature Verification}

Input: Domain Parameters, signature $(r, s)$ message $M$ and the public key of signer $Q$.

Output: accepted or rejected.

$w=s-1 \bmod n$

$u=H(M) w \bmod n$

$v=r w \bmod n$

$R=u G+v Q \in E(F q)$

Accept the signature if and only if $x(R)=r \bmod n$

\section{Encryption:}

Input: ID, params, $E$, and plain $M$.

For more information about the Conference please visit the websites: 
Output: cipher $C$. AES generate series of AES round keys from $E$ and return (AES_round_key).

Initial Step:

$\mathrm{N}_{\mathrm{b}} \leftarrow 4$ (data blocks are of 128 bits)

$\mathrm{N}_{\mathrm{r}} \leftarrow$ number of rounds in the cipher $\left(\mathrm{N}_{\mathrm{r}}=10\right.$ for AES-128)

Step 1:

State $\leftarrow$ plain

AddRoundKey (state, w $(0, \mathrm{Nb}-1))$

Step 2:

For all round do where $\{1 \leq$ round $<\mathrm{Nr}\}$

SubBytes (state)

ShiftRows (state)

MixColumns (state)

AddRoundKey (state, w (round $* \mathrm{Nb}$, (round +1$) * \mathrm{Nb}-1)$ )

End for

Step 3:

SubBytes (state)

ShiftRows (state)

AddRoundKey (state, w $[\mathrm{Nr} * \mathrm{Nb},(\mathrm{Nr}+1) * \mathrm{Nb}-1])$

cipher text $\leftarrow$ state

Step 4:

Return (cipher)

\section{Decryption:}

Input: ID, params, Cert, $E$, and cipher $C$

Output: plain $M$

Initial Step:

$\mathrm{Nb} \leftarrow 4$ (data blocks are of 128 bits)

$\mathrm{Nr} \leftarrow$ number of rounds in the cipher $(\mathrm{Nr}=10$ for AES-128)

Step 1:

State $\leftarrow$ cipher

AddRoundKey (state, $\mathrm{w}(\mathrm{Nr} * \mathrm{Nb},(\mathrm{Nr}+1) * \mathrm{Nb}-1))$

Step 2:

For all-round do where $\{\mathrm{Nr}>$ round $\geq 1\}$ decrement round by 1

InvShiftRows (state)

InvSubBytes (state)

AddRoundKey (state, w (round $* \mathrm{Nb},($ round +1$) * \mathrm{Nb}-1)$ )

InvMix Columns (state)

End for

Step 3:

InvShiftRows (state)

InvSubBytes (state)

AddRoundKey (state, w $(0, \mathrm{Nb}-1))$

Plain $\leftarrow$ state

Step 4:

Return (Plain) 
However, the algorithm of the proposed model is described in detail in Table $\mathbf{1 .}$

Table 1: The Algorithm of Proposed Model

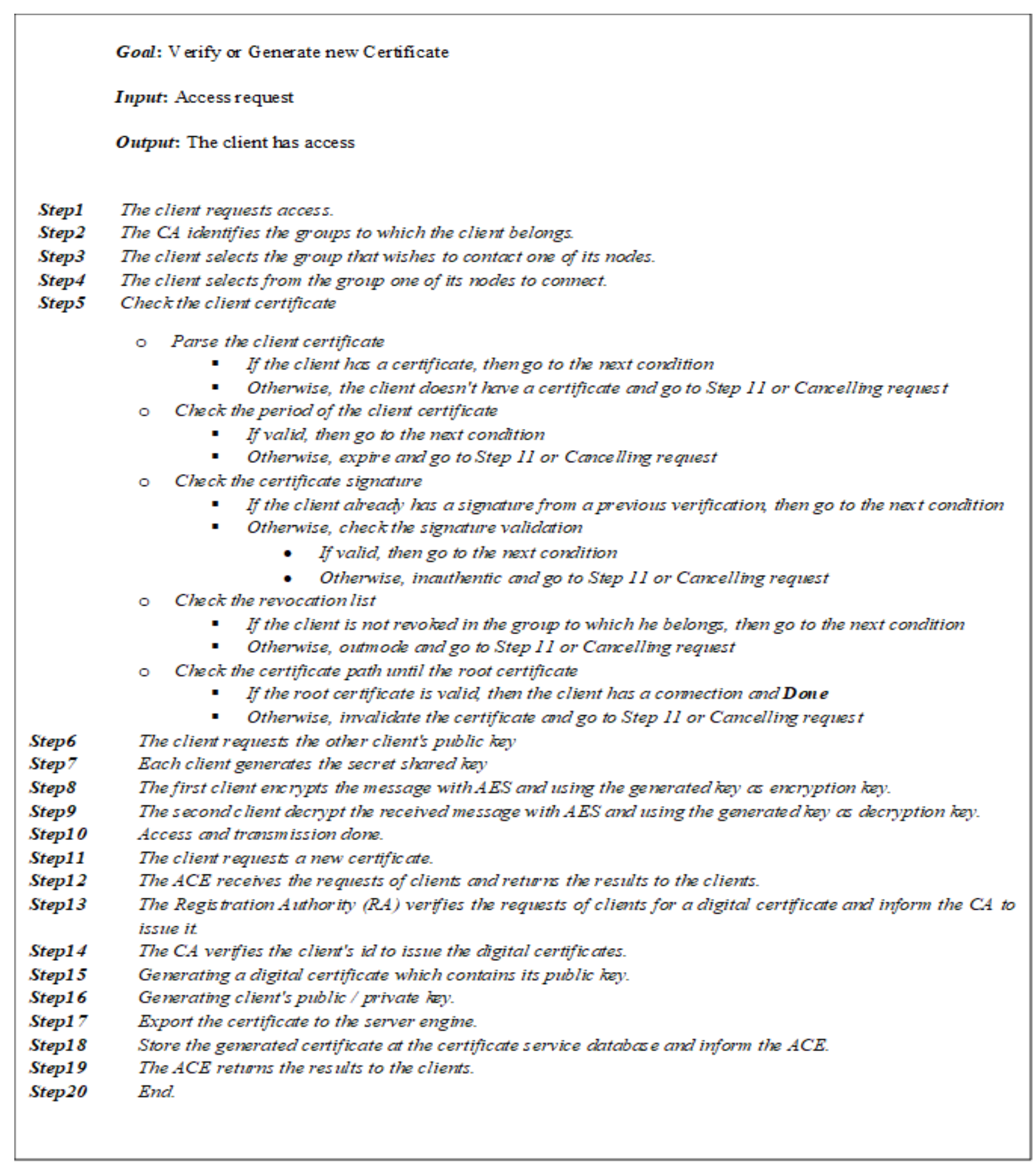

For more information about the Conference please visit the websites: 


\section{Cryptographic Tool and Dataset}

The proposed model was coded in the C\# programming language. Also, the open code library "Bouncy Castle" has been used to compose the code. This library is created by the Legion of Bouncy Castle and is a C\# usage of cryptographic algorithms.

Friendster social network and ground-truth communities have been used as a dataset for our model (Yang and Leskovec, 2015). Friendster is an online gaming network where clients can create friendship edges with each other. The dataset includes a lot of groups of nodes, each node is either linked to at least one group or is linked to other nodes that belong to different groups. The dataset contains $65,608,366$ nodes and 1,806,067,135 edges.

\section{System Analysis and Performance Security Proof}

There are two attacks against digital signatures regarding the ECDSA algorithm: a key attack wherein the adversary has a knowledge of the public key and a message attack wherein the adversary has got right of entry to signatures earlier than cracking the function. However, there are various understandings of break a digital signature: recovering the private key, producing an alternative algorithm of signature with a corresponding private key, and then forge a signature for a specific message (Goldwasser et al., 1988).

There are fundamental conditions for ECDSA to make it hard to break and have powerful security (Vaudenay, 2003):

- The discrete logarithm inside the subgroup spanned with the aid of $G$ is difficult. This makes sure it is difficult to resolve the discrete logarithm problem and consequently not possible access the private key.

- The used hash function is a one-way collision-resistant. When could not specify $m$ from $(m)=y$, we can say one-way collision-resistant. However, collision-resistant function has a little opportunity of mapping different messages to be equivalent (i.e. $H(m 1)=H(m 2))$.

- The generator for $k$ is could not predict. Else, the private key can be acquired using $k, r$, and $s$.

Concerning ECDSA, there are two major attacks that either versus the hash function used within the signature generation or against ECDLP (Johnson, Menezes, and Vanstone, 2004). If the algorithm no longer comprises the second bullet point from above, the attacker can discover a collision in the hash function by two messages, and sign a message but claim his signature on the other. ECDLP is described as solving for $d$ in $Q=d G$ within the key generation algorithm. There are common attacks versus ECDLP involving the "PohligHellman", "exhaustive seek" and "baby-Step giant-Step" algorithms. Besides these attacks, Pollard's Rho algorithm has a running time of $\left(ل_{n} \pi\right) / 2$, in which $n$ is the order of point $G$. However, this algorithm parallelized and run on $r$ various processors, hence the new running time is $(\sqrt{ } \mathrm{n} \pi) / 2 \mathrm{r}$.

For more information about the Conference please visit the websites:

http://ihicps.com/ $\quad$ C o m p u t e r |90




\section{Encryption Time Consummation}

In this section, a comparative test of the time consumption of our proposed CBE scheme against the RSA algorithm is discussed. Key generation and encryption/decryption of 40 bits' string of characters is tested based on the PC of Core i7 with Frequency 2.2GHz, RAM $8 \mathrm{~GB}$, and the operating system is Windows10 with 64bit. These comparisons show the time consumption of the key generation when using ECC of the proposed CBE scheme against RSA as shown in Table 2, and the encryption/decryption when using AES of the proposed CBE scheme against RSA algorithm as shown in Table 3.

Table 2: Time Consumption of Key Generation (ms)

\begin{tabular}{|c|c|c|}
\hline \multirow{2}{*}{ Key length } & \multicolumn{2}{|c|}{ Key pair generation } \\
\cline { 2 - 3 } & RSA & ECC \\
\hline $\mathbf{1 0 2 4} / \mathbf{1 6 0}$ & 237 & 218 \\
\hline $\mathbf{2 0 4 8} / \mathbf{2 2 4}$ & 1440 & 232 \\
\hline $\mathbf{3 0 7 2} / \mathbf{2 5 6}$ & 3377 & 234 \\
\hline $\mathbf{7 6 8 0} / \mathbf{3 8 4}$ & 108718 & 278 \\
\hline $\mathbf{1 5 3 6 0} / \mathbf{5 1 2}$ & 925284 & 375 \\
\hline
\end{tabular}

Table 3: Time Consumption of Encryption/Decryption (ms) and ( $\mu \mathrm{s})$

\begin{tabular}{|c|c|c|c|c|c|c|}
\hline \multirow{2}{*}{ Key length } & \multicolumn{2}{|c|}{ Encryption } & \multicolumn{2}{c|}{ Decryption } & \multicolumn{2}{c|}{$\begin{array}{c}\text { Total (Encryption } \\
\text { \& Decryption) }\end{array}$} \\
\cline { 2 - 7 } & RSA & AES & RSA & AES & RSA & AES \\
\hline $1024 / 160$ & $680 \mu \mathrm{s}$ & $230 \mu \mathrm{s}$ & 4 & 1 & 5 & 2 \\
\hline $2048 / 224$ & $870 \mu \mathrm{s}$ & $440 \mu \mathrm{s}$ & 16 & 2 & 17 & 3 \\
\hline $3072 / 256$ & 3 & 1 & 32 & 2 & 35 & 3 \\
\hline $7680 / 384$ & 6 & 2 & 559 & 2 & 565 & 4 \\
\hline $15360 / 512$ & 19 & 3 & 3456 & 4 & 3475 & 7 \\
\hline
\end{tabular}

Although AES is faster than RSA in encryption, it is noticeable that the gap between ECC and RSA systems grows rapidly as the key sizes increase in key pair generation. Subsequently, the RSA system is much more time-consuming than the ECC system. Hence to obtain an efficient and higher security level, especially in the big data environment, the key sizes must expand and it is advisable to select the ECC-based system.

For more information about the Conference please visit the websites: 


\section{Statistical Analysis}

Big Data has a characteristic of the variety, so it may contain images. Therefore, several tests were performed to encrypt the image using the proposed scheme. However, to resist statistical attacks, the encrypted images should possess certain random properties. Independence and uniformity are two properties that should be satisfied by any encryption scheme to ensure high resistance against statistical attacks. To prove the performance of the improved cryptosystem and verify these properties, a statistical analysis has been performed by calculating the correlation, SSIM, MSE, and histograms for the plain image and the encrypted image. Several images have been tested, and it has shown that the intensity values are good as illustrated in Table 4.

Table 4. Results of Differential Analysis

\begin{tabular}{|l|c|c|c|c|}
\hline \multirow{2}{*}{ Image } & \multicolumn{2}{|c|}{ Correlation } & SSIM & MSE \\
\cline { 2 - 4 } & Original image & Encrypted image & & \\
\hline Lena & 0.9959 & 0.0009 & 0.1061 & 0.3346 \\
\hline Airplane & 0.9948 & 0.0004 & 0.1112 & 0.3347 \\
\hline Pepper & 0.9966 & 0.0021 & 0.1067 & 0.03345 \\
\hline Tulips & 0.9981 & 0.0061 & 0.0933 & 0.3384 \\
\hline
\end{tabular}

The experimental result of the plain image and its corresponding cipher image and their histograms are shown in Figure 2. The figure shows the original plain image and its cipher image encrypted by the improving scheme. The result reveals that the improved scheme has reliable encryption and decryption effect. The histogram of the encrypted image is nearly uniformly distributed and significantly different from the respective histograms of the original images, which means that the improved algorithm has an excellent performance in resisting statistical attacks.

For more information about the Conference please visit the websites:

http://ihicps.com/ C C m p u t e r |92 


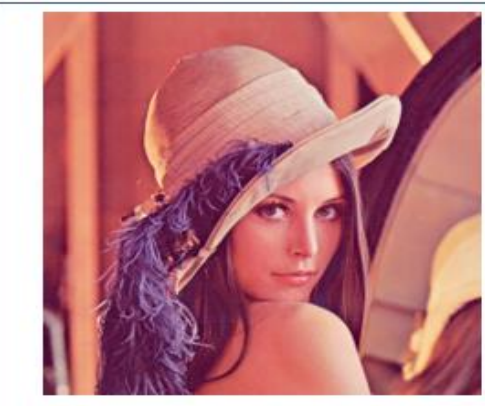

a

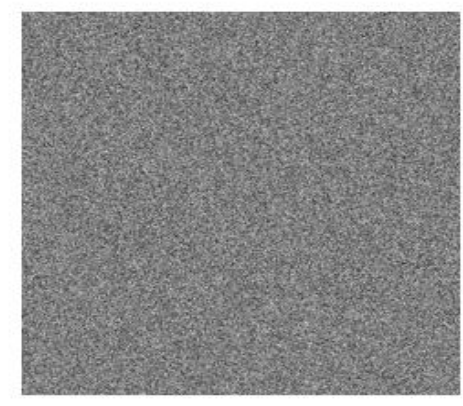

c

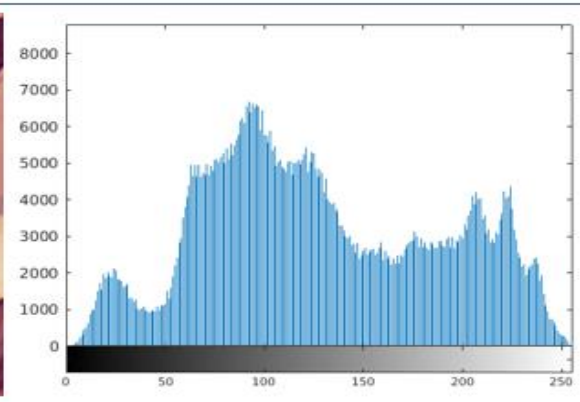

b

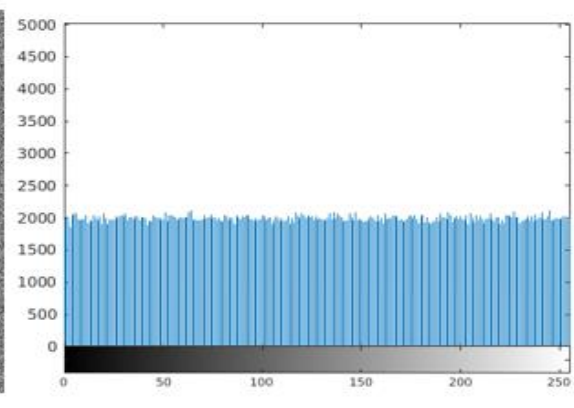

d

Figure 2. (a) Original Image (b) Histogram of Original Image (c) Encrypted Image (d) Histogram of Encrypted Image

\section{Conclusion}

An improving algorithm of Certificate-Based Encryption has been presented to using it in the Big Data environment. The system can handle the risks of the compromised CA and key escrow problem, as well as ensure the privacy of communications between clients because the key generation is done at the user level without any intervention from the certificate provider using ECDH, and hence the private key cannot be illegally obtained.

The combining of ECDSA as well ECDH will build a strong system that ensures secure communication among the entities. The key exchange process takes place after the completion of the authentication process, where the authentication is done by verifying the certificate. As a result, the key exchange operation is secured. The keys are then exchanged to generate a shared secret key, which is used as a key to encrypt and decrypt the data.

Consequently, this ensures that two-way data security each one completes the other. The certificate provider cannot decrypt the ciphertext, and at the same time, the user can not send the data without a verified certificate approved by the certificate provider. The proposed scheme tested on a big dataset of social networks. A comparison of the time consumption of the model has been tested and shown to be fast in key generation, encryption, and decryption. However, since the variety is a characteristic of big data, several tests were performed to encrypt the images using the proposed scheme and it has shown that the intensity values are acceptable.

\section{References}

1.Ahmed, H. E. H.; Kalash, H. M., ; Farag Allah, O. S. (2007). Encryption efficiency analysis and security evaluation of RC6 block cipher for digital images. 2007 International Conference on Electrical Engineering,ICEE. https://doi.org/10.1109/ICEE.2007.4287293.

2.A Shamir ,Identity Based Cryptosystem and Signature Schemes, Advances in Cryptology Proceedings of CRYPTO, 1984, 84, 7-53. Available at:

For more information about the Conference please visit the websites:

http://ihicps.com/ $\quad$ C o m p u t e r |93 
http://discovery.csc.ncsu.edu/Courses/csc774-S08/reading-assignments/shamir84.pdf.

3.Anoop, M. S. Elliptic Curve Cryptography - An Implementation Tutorial Elliptic Curve

Cryptography An Implementation Guide. $2001,1-11 . \quad$ Available at:http://www.infosecwriters.com/text_resources/pdf/Elliptic_Curve_AnnopMS.pdf.

4.Cheng, L., Liu, F. ; Yao, D. D. Enterprise data breach: causes, challenges, prevention, and future directions, Wiley Interdisciplinary Reviews: Data Mining and Knowledge Discovery, 2017, 7(5), 1-14. doi: 10.1002/widm.1211.

5.Galindo, D., Morillo, P. ; Ràfols, C. Improved certificate-based encryption in the standard model, Journal of Systems and Software, 2008, 81(7), 1218-1226. doi: 10.1016/j.jss.2007.09.009.

6.Gentry, C. Certificate-Based Encryption and the Certificate Revocation Problem, International Conference on the Theory and Applications of Cryptographic Techniques: Advances in Cryptology — EUROCRYPT, 2003, 272-293. doi: 10.1007/3-540-39200-9_17.

7.Goldwasser, S., Micali, S., ; Rivest, R. L. A digital signature scheme secure against adaptive chosen-message attacks, SIAM Journal on Computing, 1988,17(2), 281-308.

8.Daoud, A. O., Tsehayae, A. A.; Fayek, A. R. A guided evaluation of the impact of research and development partnerships on university, industry, and government. Canadian Journal of Civil Engineering, 2017,44(4),253-263.

9.Hwang, S. O. ; Le, M. H. Efficient certificate-based encryption and hierarchical certificatebased encryption schemes in the standard model, Journal of Intelligent and Fuzzy Systems, 2018, 35(6), 5971-5981. doi: 10.3233/JIFS-169838.

10. Hyla T., Maćków W., Pejaś J. Implicit and Explicit Certificates-Based Encryption Scheme. In: Saeed K., Snášel V. (eds) Computer Information Systems and Industrial Management. CISIM 2015. Lecture Notes in Computer Science, vol 8838. Springer, Berlin, Heidelberg. 2014, 651-666. doi:10.1007/978-3-662-45237-0_59.

11.Johnson, D.; Menezes, A. ; Vanstone, S. The Elliptic Curve Digital Signature Algorithm Validation System ( ECDSAVS ).2004, 56. Available at: http://cs.ucsb.edu/ koc/ccs130h/notes/ecdsa-cert.pdf.

12.Langford, Susan K. Threshold DSS signatures without a trusted party." Annual International Cryptology Conference. Springer, Berlin, Heidelberg, 1995.

13.Lehmann, E. L.; Casella, G. Theory of Point Estimation, Second Edition Springer Texts in Statistics In Design. 1998,41. https://doi.org/10.2307/1270597

14.Le, M. H., Kim, I. ; Hwang, S. O. 'Efficient certificate-based encryption schemes without pairing', Security and Communication Networks. 2016, 9(18), 5376-5391. doi: 10.1002/sec. 1703 .

15.Liu, J. K. ; Zhou, J. Efficient certificate-based encryption in the standard model, Lecture Notes in Computer Science (including subseries Lecture Notes in Artificial Intelligence and Lecture Notes in Bioinformatics), 5229 LNCS, 2008, 144-155. doi: 10.1007/978-3-54085855-3_10.

16.Lu, Y. ; Li, J. Efficient certificate-based encryption scheme secure against key replacement attacks in the standard model, Journal of Information Science and Engineering, 2014,30(5), $1553-1568$.

17.Lu, Y., Li, J. and Xiao, J. (2008) Generic construction of certificate-based encryption, Proceedings of the 9th International Conference for Young Computer Scientists, ICYCS For more information about the Conference please visit the websites: 
2008,1589-1594. doi: 10.1109/ICYCS.2008.11.

18.S. Vanstone, Responses to NIST's Proposal, Communications of the ACM, 35, July 1992, 50-52 (communicated by John Anderson).

19.Yang, J., ; Leskovec, J. Defining and evaluating network communities based on groundtruth. Knowledge and Information Systems, 2015,42(1), 181-213.Retrievedfrom https://snap.stanford.edu/data/com-Friendster.html

20.Vaudenay, S. (2002) The security of DSA and ECDSA: Bypassing the standard elliptic curve certification scheme, Public Key Cryptography - PKC 2003, 2567, 309-323. 